\title{
THE COHOMOLOGY RING OF A COMPACT LIE GROUP WITH BI-INVARIANT METRIC
}

\section{A. L. MAYER}

I. Introduction. In this note we shall show that the adjoint operation* obtained from a bi-invariant riemannian metric on a compact Lie group induces an isomorphism between the cup and Pontrjagin products on the cohomology ring. This fact is easily and directly verifiable in the case of a torus, where, as we shall show elsewhere, it has interesting applications to the classical theory of abelian varieties; in fact, it motivates a definition of $*$ on the numerical equivalence ring of an abstract polarized abelian variety.

II. Algebraic preliminaries. Let $E$ be an $n$-dimensional vector space over $R, \Lambda^{p}(E)$ the $p$-fold exterior product, and $\tilde{E}, \Lambda^{p}(E)^{\sim}$, their respective dual spaces. There is a canonical isomorphism $i_{p}: \Lambda^{p}(E)^{\sim} \rightarrow \Lambda^{p}(\widetilde{E})$. An orientation of $E$ is an isomorphism $\epsilon: \Lambda^{n}(E)$ $\cong R$. It gives rise to a dual orientation $\tilde{\epsilon}: \Lambda^{n}(\tilde{E}) \cong R$, and we denote the fundamental $n$-vector and $n$-covector by $e=\epsilon^{-1}(1)$ and $\tilde{e}=\tilde{\epsilon}^{-1}(1)$, respectively. One defines an isomorphism $j_{p}: \Lambda^{p}(E) \rightarrow \Lambda^{n-p}(E)^{\sim}$ by letting $j_{p}(\alpha)(\beta)=\epsilon(\alpha \wedge \beta)$. This gives an isomorphism $k_{p}=i_{n-p} \circ j_{p}$ : $\Lambda^{p}(E) \cong \bigwedge^{n-p}(\tilde{E})$. For $\alpha \in \bigwedge^{p}(E), \beta \in \Lambda^{q}(E)$, let $\alpha \bigvee \beta=k^{-1}(k \alpha \wedge k \beta)$ $\in \Lambda^{p+q-n}(E)$, and for $\tilde{\beta} \in \Lambda^{q}(\widetilde{E}), \alpha \pi \widetilde{\beta}=\alpha \bigvee^{-1}(\widetilde{\beta}) \in \Lambda^{p-q}(E)$.

The composition map $T: E \oplus E \rightarrow E$, sending $\alpha \oplus \beta$ into $\alpha+\beta$, can be uniquely extended to an algebra homomorphism $\left\{T^{p}\right\}, T^{p}: \Lambda^{p}(E \oplus E)$ $\rightarrow \Lambda^{p}(E)$. Since $\Lambda^{p}(E \oplus E) \cong \oplus_{r+s=p} \Lambda^{r}(E) \otimes \Lambda^{s}(E)$, we can define, for $\alpha \in \Lambda^{r}(E)$ and $\beta \in \Lambda^{\cdot}(E), T^{r+s}(\alpha \otimes \beta) \in \Lambda^{r+s}(E)$, and a simple computation shows this to equal $\alpha \wedge \beta$. Also, $T: E \oplus E \rightarrow E$ may be dualized to give $\widetilde{T}: \tilde{E} \rightarrow \tilde{E} \oplus \widetilde{E}$, which extends uniquely to an algebra homomorphism $\left\{\tilde{T}^{p}\right\}, T^{p}: \Lambda^{p}(\tilde{E}) \rightarrow \bigwedge^{p}(\tilde{E} \oplus \tilde{E})$. Let $\tilde{e}^{\prime}=T^{n}(\tilde{e}) \in \Lambda^{n}(\tilde{E} \oplus \tilde{E})$. Given $\alpha \in \Lambda^{p}(E), \quad \beta \in \Lambda^{q}(E)$, we have $\alpha \otimes \beta \in \Lambda^{p+q}(E \oplus E)$ and $\alpha \otimes \beta \pi \tilde{e}^{\prime} \in \Lambda^{p+q-n}(E \oplus E)$. An easy computation shows that $T^{p+q-n}\left(\alpha \otimes \beta \pi \tilde{e}^{\prime}\right)=\alpha \bigvee \beta$.

A quadratic form on $E$ is an isomorphism $\phi: E \rightarrow \tilde{E}$, extendable uniquely to an algebra isomorphism $\left\{\phi^{p}\right\}, \phi^{p}: \Lambda^{p}(E) \rightarrow \Lambda^{p}(\tilde{E})$. We define ${ }^{*}: \bigwedge^{p}(E) \rightarrow \bigwedge^{n-p}(E)$ by ${ }^{*} \alpha=k^{-1} \phi \alpha$. Note that ${ }^{*}(\alpha \wedge \beta)$ $=k^{-1}(\phi \alpha \wedge \phi \beta)=k^{-1}\left(k k^{-1} \phi \alpha \wedge k k^{-1} \phi \beta\right)=k^{-1}\left(k^{*} \alpha \wedge k^{*} \beta\right)={ }^{*} \alpha \bigvee^{*} \beta$. So * is an isomorphism of the $\wedge$-algebra onto the $\vee$-algebra; if $\phi$ is unitary, i.e., ${ }^{*} 1=e$, the map is unit preserving.

Received by the editors February 2, 1963. 
Needless to say, the above considerations extend to the case where $E$ is a vector bundle over a manifold $M$; e.g., if $E$ is the cotangent bundle of an oriented manifold, we recover the familiar * of WeitzenbackHodge.

III. Integration along the fibre. Let $G$ be a compact oriented Lie group with dual Lie algebra $E$ (whose orientation is $\epsilon$ ), and let $\Phi: Y^{k} \rightarrow X^{m}$ be a principal fibre bundle with group $G, X$ and $Y$ being compact and having orientations compatible with that of $G$ (with respect to the local product structure). The orientation of $E$ gives $G$ a unique left-invariant Haar measure. For all $x \in X$, there is a homeomorphism $\psi_{x}: G \rightarrow \Phi^{-1}(x)$, sending the invariant $n$-vector $\tilde{e}$ onto an invariant $n$-vector $\tilde{e}_{0}$ along the fibre. Let $\alpha$ be a $p$-form on $Y$; we define a $(p-n)$-form ${ }_{*} \Phi(\alpha)$ on $X$ by integrating along the fibre. Precisely, $\alpha \pi \tilde{e}_{0}$, along the fibre $\Phi^{-1}(x)$ is a form annihilating all vectors tangent to the fibre; hence it may be integrated, by integrating its coefficients with respect to the Haar measure, to give a $(p-n)$-form ${ }_{*} \Phi(\alpha)_{x}$ at $x$. The local product structure and the invariance of the measure guarantee that $* \Phi(\alpha)$ is well defined and differentiable, and it is easy to check that ${ }_{*} \Phi(\alpha)$ is closed if $\alpha$ is.

Let $y$ be a $p$-cohomology class in $Y$, the de Rham class of a form $\alpha$. Its Poincaré dual class $P y \in H_{k-p}(Y, R)$ is defined as a linear functional on $H^{k-p}(Y, R)$ by $P y(z)=\int_{Y} \alpha \wedge \beta, z$ being the de Rham class of a form $\beta$. The map $\Phi$ induces a map $\Phi_{*}: H_{k-p}(Y, R) \rightarrow H_{k-p}(X, R)$ by letting $\Phi_{*}(P y)(w)=\int_{Y} \alpha \wedge \Phi^{*} \gamma$, where $w$ is the de Rham class of a $(k-p)$-form $\gamma$ on $X$. On the other hand, the Poincaré dual class of ${ }_{*} \Phi(\alpha)$ is defined as a functional on $H^{k-p}(X, R)$ by sending the de Rham class $w$ of a form $\gamma$ into $\int_{X *} \Phi(\alpha) \wedge \gamma$. A partition of unity and Fubini's theorem show immediately that $P\left[{ }_{*} \Phi(\alpha)\right]=\Phi_{*}(P y), y$ again being the class of $\alpha$. Hence $* \Phi$ induces on the de Rham cohomology the well-known Gysin homomorphism.

IV. The convolution product. Now let $X=G, Y=G \times G$ and $\Phi$ be the composition map. Further, let $\pi_{1}$ and $\pi_{2}$ be the projections of $G \times G$ onto its factors. If $\alpha$ is a $p$-form, and $\beta$ a $q$-form, on $G$, the $(p+q-n)$-form $\alpha \odot \beta={ }_{*} \Phi\left(\pi_{1}{ }^{*} \alpha \wedge \pi_{2}{ }^{*} \beta\right)$ will be called the convolution of $\alpha$ and $\beta$. (If $p=q=n, \alpha=f e$ and $\beta=g e$, then $\alpha \beta \beta=h e$, and it is clear that the function $h$ is the convolution, in the usual sense, of the functions $f$ and $g$.) From the last remarks in $\S I I I$, it is clear that the convolution algebra on closed forms induces on the cohomology of $G$ the algebra structure obtained by transposing (via Poincaré duality) the Pontrjagin algebra on homology to cohomology.

Now let $\alpha$ be right invariant, and $\beta$ left invariant. Then $\pi_{1}^{*} \alpha$ 
$\wedge \pi \beta_{2}{ }^{*} \pi \tilde{e}_{0}$ is invariant along the fibre $\Phi^{-1}(1)$ so the value of $\alpha \bullet \beta$ at 1 is merely the image under the canonical map $\wedge^{p+q-n}(E \oplus E)$ $\rightarrow \wedge^{p+q-n}(E)$ of the value of $\pi_{1}^{*} \alpha \wedge \pi_{2}{ }^{*} \beta \tilde{e} \pi_{0}$ at $1 \times 1$. (We are using the fact that the map $\psi_{1}: G \rightarrow G \times G$ induces, on the dual Lie algebra, the canonical map of composition.) By the remarks in II, $\alpha \bigcirc \beta$ evaluated at 1 is thus (the value of $\alpha$ at 1$) \bigvee($ the value of $\beta$ at 1 ).

Now assume that both $\alpha$ and $\beta$ are bi-invariant. Then $\pi_{1}{ }^{*} \alpha \wedge \pi_{2}{ }^{*} \beta$ is bi-invariant on $G \times G$; invariant, in particular, under the liftings via $\Phi$ of both right and left translations on $G$. Hence, $\alpha \bullet \beta$ is bi-invariant, and so equals $\alpha \bigvee \beta$.

V. Conclusion of the proof. We may map $H^{*}(G, R)$ into $\Lambda^{*}(E)$ by sending each class into the unique bi-invariant form it contains, evaluated at 1. It is well known that this is a homomorphism of the cup-algebra into the $\wedge$-algebra, and we have shown above that it is also a homomorphism of the (transposed) Pontrjagin algebra into the $\checkmark$-algebra.

Now, let $\phi$ be a bi-invariant riemannian metric on $G$. (One is easily constructed by integrating a positive-definite quadratic form on $\widetilde{E}$ under the adjoint group, and then translating.) Then (e.g., since biinvariant forms are harmonic) the action of ${ }^{*}$ on $\Lambda^{*}(E)$ is compatible with the action of $*$ on cohomology. Letting $\oplus$ denote the (cohomological) Pontrjagin product, we may combine this last remark with that at the end of $\S I$ II to assert that, for any cohomology classes $x$ and $y$ on $G,{ }^{*}(x \cup y)=\left({ }^{*} x\right) \oplus\left({ }^{*} y\right)$.

\section{Columbia University}

